\title{
The Biosynthesis by Brain Microsomes of Cerebrosides Containing Nonhydroxy Fatty Acids, 2
}

\author{
PIERRE MORELL, ELVIRA COSTANTINO-CECCARINI, \\ AND NORMAN S. RADIN
}

\begin{abstract}
Saul R. Korey Department of Neurology and the Department of Biochemistry, Albert Einstein College of Medicine, Bronx, New York 10461 and The Mental Health Research Institute, University of Michigan, Ann Arbor, Michigan 48104
\end{abstract}

Received July 22, 1970; accepted Sept. 17, 1970

\begin{abstract}
Incubation of microsomes from mouse brain with UDP-gal, a long-chain base, and ${ }^{14} \mathrm{C}$-fatty acyl-CoA resulted in formation of NFA-cerebroside labeled in the fatty acid residue. The rate of synthesis was ten times that of the system lacking UDP-gal or long chain base. The incubation system also formed NFA-ceramide, at a much higher rate. Stearoyl- and lignoceroyl-CoA were much more effective in forming ceramide and cerebroside than palmitoyl- and oleoyl-CoA, a correlation which suggests that ceramide is an intermediate in ecrebroside biosynthesis.

When NFA-ceramide, together with a phospholipid mixture, was incubated with labeled UDP-gal, NFA-cerebroside was formed. Utilization of the exogenous ceramide was demonstrated by a number of chromatographic procedures. Under the same conditions, but with labeled UDP-gle, the corresponding glucose-containing cerebroside was formed, at a somewhat higher rate.

The organic synthesis of labeled lignoceric acid and lignoceroyl D-sphingosine is described. Also described is a simplified method for large-scale preparation of purified $D$-sphingosine. The labeled ceramide was utilized by the microsomes for the formation of galactosyl lignoceroyl sphingusine.

Under a variety of conditions the microsomes failed to convert psychosine and labeled acyl-CoA to cerebroside.
\end{abstract}

The major cerebroside of brain consists of a long chain base (usually sphingosine

1 This study was supported by Grants NS 03356 , NS 09094, and NS 03192 from the National Institute of Neurological Diseases and Stroke, U.S. Public Health Service. Part of this work was done while one of us (P.M.) was supported by Training Grant MH-07417 from the National Institute of Mental Health.

${ }^{2}$ Abbreviations used: HFA, 2-hydroxy fatty acid; NFA, nonhydroxy fatty acid; TLC, thinlayer chromatography. The term "long-chain base" refers here to sphingosine or dihydrosphingosine. Psychosine is galactosyl long-chain base; ceramide is $N$-acyl long-chain base. The term "cerebroside" is generally used here to mean galactosyl ceramide; "glucocerebroside" is glucosyl ceramide. The standard numerical abbreviations are used for fatty acids: 18:0 = stearate, 24:0 = lignocerate. or dihydrosphingosine) with its primary hydroxyl group in a $\beta$-glycosidic linkage to galactose and the basic group in amide linkage with a fatty acid. This fatty acid can be nonhydroxy fatty acid (in NFAcerebroside) or a hydroxy fatty acid (HFAcerebroside). Presently available evidence indicates that UDP-gal (1) and acyl-CoA are the high-energy donors of the hexose and fatty acyl moieties, respectively. Two possible biosynthetic routes are:

(a) Galactosylation of a long-chain base followed by acylation:

$$
\begin{gathered}
\text { long-chain base }+ \text { UDP-gal } \\
\qquad \begin{aligned}
\rightarrow \text { psychosine }+ \text { UDP } \\
\text { psychosine }+ \text { acyl-CoA } \\
\rightarrow \text { cerebroside }+\mathrm{Co \Lambda}
\end{aligned}
\end{gathered}
$$


(b) or the reverse sequence:

long-chain base + acyl-CoA

ceramide + UDP-gal

$$
\rightarrow \text { ceramide }+\mathrm{CoA}
$$

$$
\rightarrow \text { cerebroside + UDP }
$$

Evidence for scheme (a) is the demonstration of psychosine formation by Cleland and Kennedy (2), a finding confirmed by other investigators. A preliminary communication reporting the acylation step (Eq. 2) has been published by Brady (3).

Results supporting scheme (b) include the demonstration of Eq. 3 by Sribney (4). This acylation has been characterized with respect to specificity for fatty acyl-CoA and long-chain base precursors by Morell and Radin (5). NFA-ceramide appears to be the precursor for formation of glucocerebroside (6). We (7), as well as Basu, Schultz, and Basu (8) have demonstrated in vitro the galactosylation of HFA-ceramide, to form HFA-cerebroside. The same reaction has been confirmed in kidney by Coles and Gray (9).

In this investigation we developed conditions under which microsomal particles from young mouse brain could be used to demonstrate synthesis of NFA-cerebroside from its three precursors, long-chain base, acyl-CoA, and UDP-gal. Having established these conditions, we attempted to differentiate between the two possible reaction sequences (a) and (b) by study of the two terminal reactions. While reactions (1) and (3) could be readily confirmed, psychosine acylation could not be observed. However, the galactosylation of NFA-ceramide could be demonstrated although the rate of reaction was relatively low. An important factor in eliciting the reaction was the use of a phospholipid preparation as a stimulating adjunct.

\section{MATERIALS AND METHODS}

Materials. Most of the materials and standards used were obtained as previously described $(5,7$, 10). ${ }^{14} \mathrm{C}$ UDP-gal and ${ }^{14} \mathrm{C}$-UDP-gle were obtained from New England Nuclear Corp. (Boston, Mass.). A preparation of beef heart "lecithin" was obtaincd from Sylvana Chemical Company, Orange, New Jersey. (TLC showed this contains also some sphingomyelin and lysolecithin.) DL-erythrodihydrosphingosine were purchased from Miles Laboratories. (Elkhart, Indiana). Swiss-Webster mice was obtained from Carworth, New City, New York.

Synthetic procedures. Radioactive acyl-CoA's were synthesized by the mixed anhydride method (11) with modifications for the synthesis of lignoceroyl-CoA (5). A mixture of sphingolipids was prepared from beef spinal cord lipids by the method of Radin and Brown (12). Ceramides were prepared by the method of Carter et al. (13) and fractionated into NFA- and HFA-ceramides by column chromatography as described previously (7). Stearoyl and lignoceroyl D-erythro-sphingosine were synthesized from the free base and acyl chloride (14).

Labeled lignoceric acid was made by decarboxylating unlabeled silver lignocerate with bromine (Hunsdiecker reaction), condensing the tricosanyl bromide with labeled cyanide, and hydrolyzing the nitrile $(15,16)$. The poor solubility of the high molecular weight compounds involved and the scarcity of detail in the literature on these reactions necessitated some experimentation before the steps could be made to go conveniently. Thus, we present the procedures in some detail.

Two mmoles of lignoceric acid (Hormel Institute, Lipid Preparation Laboratory) were dissolved in $16 \mathrm{ml}$ of absolute alcohol containing 2 mmoles of Primene 81-R (a high molecular weight branched primary amine from Rohm and Haas Co.). The solution of the salt was heated under reflux while stirring with a spin bar, and water was added in portions until turbidity appeared $(10 \mathrm{ml})$. The mixture was cleared with $1.5 \mathrm{ml}$ absolute alcohol, and 4 mmoles of silver nitrate were added in $6 \mathrm{ml}$ of water and $10.5 \mathrm{ml}$ of absolute alcohol. The silver salt precipitated immediately, and heating had to be stopped promptly to prevent foaming. Stirring was continued while the flask cooled. The mixture was filtered with a pressure funnel (a sintered-glass funnel sealed at the top to a ball joint, through which air pressure could be applied). The precipitate was rinsed with alcoholwater 1:1 until the filtrate showed no precipitate with $\mathrm{HCl}$. The funnel and sample were dried in vacuum over $\mathrm{P}_{2} \mathrm{O}_{5}$ for a prolonged period. The yield of silver lignocerate was $91 \%$.

The silver salt ( 1.63 mmoles) was transferred to a refluxing apparatus consisting of a test tube sealed to a small condenser. Carbon tetrachloride $(5.2 \mathrm{ml})$ was added, and a thick slurry was formed by immersing the tube in an ultrasonic bath. After addition of $0.445 \mathrm{ml}$ of bromine-carbon tetrachloride (1:4) the tube was dipped into a hot oil bath sufficiently to maintain vigorous bubbling. To maintain the anhydrous conditions eritical for this reaction the bromine and carbon tetrachloride were dried before use (with sulfuric acid and phosphorus pentoxide, respertively) and the top of the condenser was covered with Saran film. Heating 
was continued until refluxing solvent could be seen on the walls of the water-cooled condenser. The mixture was cooled, transferred to a large flask with carbon tetrachloride, mixed with excess $\mathrm{NaHSO}_{3}$ in water to destroy residual bromine, and filtered by pressure through $1 \mathrm{~g}$ of Celite. The lower layer was washed with water and evaporated to dryness, giving $651 \mathrm{mg}$ of tricosanyl bromide (99\% yield).

The bromide, on examination by TLC (using hexane as developer and alkaline bromthymol blue as detector), showed good agreement in $R_{F}$ with docosanyl bromide standard, which moved more slowly than mineral oil. Traces of six polar impurities were visible. The bromide was passed through a column in hexane ( $2 \mathrm{~g}$ of silica gel, Unisil, Clarkson Chemical Co.), but the low solubility of the bromide necessitated the use of large volumes and a warm column. Because of this, the purification was incomplete and two trace impurities could be seen in the product. It would be better to use somewhat more silica gel.

The reaction with cyanide was based on the procedure of Bergström et al. (17). One mmole of the alkyl bromide was transferred to a refluxing apparatus and to this was added 0.75 mmoles of $\mathrm{K}^{14} \mathrm{CN}(3 \mathrm{mCi})$ in $0.27 \mathrm{ml}$ water, rinsed in with 4.5 $\mathrm{ml}$ of $95 \%$ alcohol. Two phases were visible and refluxing $17 \mathrm{hr}$ did not change this. An additional $1 \mathrm{ml}$ of alcohol was added, and refluxing was continued for a total of $48 \mathrm{hr}$; the mixture was now uniformly turbid.

The nitrile was hydrolyzed by adding $1.2 \mathrm{~g} \mathrm{KOH}$ and refluxing $24 \mathrm{hr}$. The product, potassium lignocerate, was transferred to a large test tube with hot alcohol and acidification with $\mathrm{HCl}$, and extraction into hexane was carried out. A spill occurred at this stage so the ${ }^{14} \mathrm{C}$ yield cannot be calculated, but it is estimated to be over $80 \%$. The labeled lignoceric acid was purified with a Unisil column, the excess bromide being eluted with hexanebenzene 2:1 and the acid, with 1:1 mixture. Purity of the product was demonstrated by TLC with hexane-ether-acetic acid (85:15:4). The single radioactive spot observed by radioautography coincided with a lignoceric acid standard.

D-Sphingosine was prepared from crude longchain bases by a simplification of the method of Barenholz and Gatt (18). A column $2.1 \mathrm{~m} \times 47 \mathrm{~mm}$ was loaded with $1750 \mathrm{~g}$ of silica gel (Merck No. 7734 ) in $4500 \mathrm{ml}$ of chloroform-methanol $1: 1$, then rinsed with $2000 \mathrm{ml}$ of chloroform. The column was loaded with $3.5 \mathrm{~g}$ crude sphingosine in $800 \mathrm{ml}$ of chloroform-methanol-2 $\mathrm{x}$ ammonium hydroxide 90:10:1. The sample was rinsed in with $950 \mathrm{ml}$ more of the same mixture. Elution was carried out with 12 liters of a similar mixture (75:25:2.5) at 900 $\mathrm{ml} / \mathrm{hr}$, collecting 600-ml fractions. Aliquots of the column fractions were flash evaporated (19) and characterized by TLC (chloroform-methanolwater-concentrated ammonium hydroxide 140: $35: 2: 2$ ).

This simplified separation results in some fractions contaminated with threo-sphingosine, which are saved for future purification. The fractions which appeared pure were evaporated to dryness as above, pooled, and lyophilized: yield, $1.43 \mathrm{~g}$. Slight darkening takes place on storage, probably due to contamination with material from the silica gel, and we store the sphingosine in the cold room to minimize this.

Analytical methods. TLC was carried out on 0.5-mm layers of Silica Gel G (Brinkmann Instruments, Westbury, N.Y.) and plates were activated for $90 \mathrm{~min}$ at $105^{\circ}$. The plates were stored in a desiccator and reactivated for $30 \mathrm{~min}$ immediately before use. Cerebrosides were separated into four classes (7), medium-chain and long-chain NFAcerebrosides and medium-chain and long-chain HFA-cerebrosides by two developments with chloroform-methanol-water (144:25:2.8). Plates were dried at least $20 \mathrm{~min}$ at $50^{\circ}$ and allowed to cool in a vacuum desiccator between TLC runs. This system also separated glucocerebroside into two classes, medium-chain and long-chain NFAglucocerebroside (20). If incorporation of radioactive fatty acid from acyl-CoA into cerebroside was being assayed, a third chromatographic development with chloroform-methanol-acetic acid (90:2:8) was included. This solvent moved all of the radioactive free fatty acid to the solvent front and eliminated overlap of trace amounts of fatty acid in the cerebroside region. Phospholipids were separated by TLC with chloroformmethanol-concentrated ammonium hydroxide $(14: 6: 1)$. Cerebroside was separated from glucocerebroside by chromatography on TLC plates containing borate $(21,22)$ with chloroformmethanol-water-ammonium hydroxide (35:10: 5:1). Lipids were visualized with iodine vapor. Radioactivity on TLC plates was located by radioautography on Kodak No-Screen X-ray film. To quantitate radioactivity, zones of silica gel were scraped and collected in scintillation vials. To each vial was added $0.5 \mathrm{ml}$ of water and $10 \mathrm{ml}$ of a solution consisting of $15.4 \mathrm{~g}$ of Permablend 111 (Packard Instrument Co.), 2.3 liters of toluene, and $600 \mathrm{ml}$ of Triton X-100 (Rohm and Haas, Philadelphia, Pa.).

Assays for cerebroside biosynthesis. Mice (17-22 days old) were decapitated, brains were removed, and a microsomal fraction was prepared as described previously (5). The microsomal pellet was resuspended in $0.25 \mathrm{M}$ sucrose to a final volume of $0.6 \mathrm{ml} / \mathrm{g}$ initial wet weight of brain. In our first experiments we studied the formation of cerebro- 
side from long-chain base, UDP-gal, and acylCoA. The lipid substrate, $0.25 \mathrm{mg}$ of $\mathrm{D}$-sphingosine or DL-dihydrosphingosine, was evaporated onto 25 $\mathrm{mg}$ of diatomaceous earth (Celite Analytical Filter Aid) from chloroform-methanol (2:1) (7). To this was added $0.6 \mu$ mole of UDP-gal, $0.5 \mu$ mole of dithiothreitol, and $1 \mu$ mole of ATP (neutralized). The tube also contained $3.5-4.0 \mathrm{mg}$ of microsomal protein and was buffered by the addition of $25 \mu$ moles of Tris- $\mathrm{HCl}$ (added as a $1 \mathrm{M}$ solution at $\mathrm{pH}$ 7.4). Radioactive stearoyl- or lignoceroyl-CoA $(0.08 \mu \mathrm{mole}, \mathrm{sp}$ act $2500 \mathrm{cpm} / \mathrm{nmole})$ was added last. This incubation mixture, in a total volume of $\mathbf{0 . 5}$ $\mathrm{ml}$ in a stoppered test tube, was shaken vigorously in a water bath at $34^{\circ}$ for $120 \mathrm{~min}$.

The incubations were terminated by the addition of $9.5 \mathrm{ml}$ of chloroform-methanol (2:1), the precipitated protein was removed by filtration through a sintered-glass funnel, and the reaction tube and funnel were rinsed with $1 \mathrm{ml}$ of chloroform-methanol $(2: 1)$. This was followed by addition of $2.3 \mathrm{ml}$ of $1 \mathrm{M} \mathrm{KCl}$ and centrifugation to separate the resultant layers. The lower phase was washed twice with $5 \mathrm{ml}$ of water-methanol (1:1) containing $0.5 \mathrm{M} \mathrm{KCl}$, and once with $5 \mathrm{ml}$ of water-methanol (1:1). The solvent layer was evaporated under nitrogen, a sphingolipid mixture added as carrier, and radioactive lipids separated from each other by TLC. The radioactive bands were located by radioautography and the band in the cerebroside region was scraped and counted.

In further experiments cerebroside formed by the galactosylation of exogenous NFA-ceramide was determined. In preparation for these incubations $0.25 \mathrm{mg}$ of NFA-ceramide and $0.25 \mathrm{mg}$ of "lecithin" were co-evaporated onto $25 \mathrm{mg}$ of Celite. Other components included $0.15 \mu$ mole of dithiothreitol, $0.3 \mu$ mole of $\mathrm{MgCl}_{2}, 0.15 \mu \mathrm{mole}$ of EDTA, and $0.3 \mu$ mole of ATP. The tube also contained $0.70-0.75 \mathrm{mg}$ of microsomal protein and 7.5 $\mu$ mole of Tris- $\mathrm{HCl}$ ( $\mathrm{pH} 7.4$ ). Radioactive UDP-gal (12.5 nmoles at a specific activity of $10 \mu \mathrm{Ci} / \mu$ mole) was added last. The reaction mixture, total volume $0.13 \mathrm{ml}$, was incubated as above. The incubation was terminated with $2.4 \mathrm{ml}$ of chloroform-methanol (2:1), filtered, and the tube rinsed with $0.5 \mathrm{ml}$ of the same solvent. Two phases were formed by the addition of $0.6 \mathrm{ml}$ of $1 \mathrm{~m} \mathrm{KCl}$ and after centrif $\mathrm{u}$ gation and aspiration of the upper phase, the lower phase was washed once with $1.4 \mathrm{ml}$ of watermethanol (1:1) containing $0.5 \mathrm{M} \mathrm{KCl}$ and once with $1.4 \mathrm{ml}$ of water-methanol (1:1). The lower layer was evaporated and radioactive lipids separated by TLC. Bands correspnding to long-chain NFAcerebroside and to medium-chain NFA-cerebroside were seraped separately and radioactivity determined.

In some experiments ${ }^{14} \mathrm{C}-\mathrm{UDP}$-glc (12.5 nmoles at $10 \mu \mathrm{Ci} / \mu$ mole) was substituted for the UDP-gal. The assay was conducted in a manner identical to that described above, except glucocerebroside was added as carrier before separation by TLC. Bands corresponding to long-chain and mediumchain NFA-glucocerebroside were collected separately.

\section{RESULTS}

\section{Formation of NFA-Cerebroside from Long- Chain Base, UDP-gal, and Acyl-CoA}

Characterization of the product. Incubation of the brain microsomes with all three precursors gives rise to the expected NFAcerebrosides, as shown in Fig. 1. Labeled cerebroside is not obtained when longchain base is omitted (lane 1), showing the need for added base. The $R_{F}$ value of each radioactive cerebroside band is evidence that the added precursors were the ones actually utilized by the enzyme preparation, rather than endogenous materials. This is seen by comparing the standard cerebroside mixture (lane 6) with the two major cerebrosides, made from labeled lignocerate and stearate and the major long-chain base, sphingosine. These radioactive bands (lanes 2 and 4 ) match the carrier and standard (spots $a$ and $b$ ) perfectly. We have previously demonstrated (7) that in this standard the upper two bands consist mainly of NFA-cerebrosides; band (a) contains long-chain (primarily $\mathrm{C}_{24}$ ) fatty acids in the $N$-acyl position while (b) has medium chain (primarily stearate) $N$-acyl fatty acids. The lower bands consist of HFA-cerebrosides and contain long- (c) and medium-chain (d) $N$-acyl HFA.

The cerebrosides made from the saturated base (lanes 3 and 5) move slightly faster than the corresponding unsaturated cerebroside standards. This is to be expected from our observations with synthetic and biosynthesized ceramides (5), which demonstrated that ceramides containing dihydrosphingosine migrate more rapidly on TLC plates than do the sphingosine analogs.

The radioactive products were further characterized by subjecting them to conditions of alkaline methanolysis [after recovery from the TLC powder] (23) and rechromatographing them with the same solvent. No change in position was observed, 


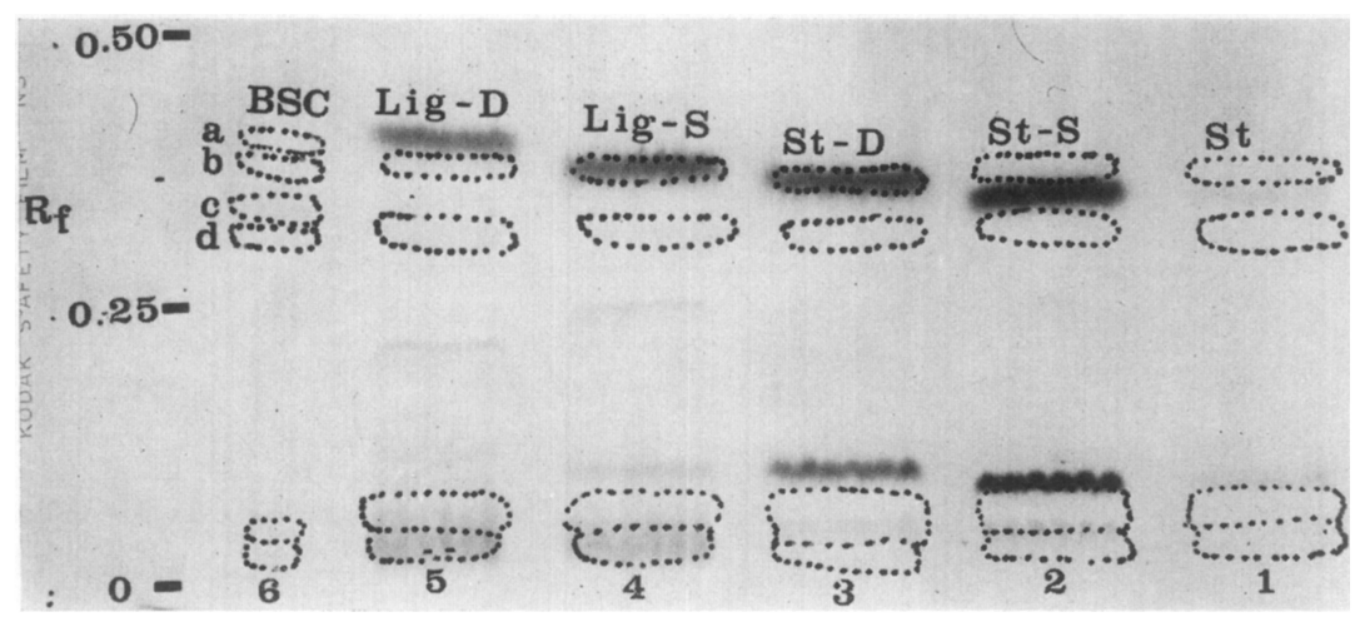

FIG. 1. Formation of cerebroside from radioactive acyl-CoA, long-chain base, and UDP-gal. Incubation conditions as described in Methods. Each tube contained UDP-gal and the following ${ }^{14} \mathrm{C}$-acyl-CoA and long-chain base components: (1) stearoyl-CoA and no long-chain base; (2) stearoyl-CoA and sphingosine; (3) stearoyl-CoA and dihydrosphingosine; (4) lignoceroyl-CoA and sphingosine; (5) lignoceroyl-CoA and dihydrosphingosine. The stearoyl-CoA had a specific activity of $2500 \mathrm{cpm} / \mathrm{nmole}$ and the lignoceroyl-CoA, 5000 $\mathrm{cpm} / \mathrm{nmole}$. Total lipids were isolated and chromatographed in the corresponding lane as described in Methods. A standard mixture of sphingolipids was run in lane 6 and carrier sphingolipids were included in each lane. The stippled lines represent lipids visualized with iodine vapors. Spots (b) and (d) although visible in each lane, were omitted for the sake of clarity.

showing that the fatty acid moieties were present in amide linkage. When the recovered cerebrosides were rechromatographed on borate-containing plates, the radioactivity appeared in the expected positions, showing that the carbohydrate moiety was galactose, rather than glucose. Acid methanolysis (24) of the purified cerebrosides yielded methyl esters containing over $95 \%$ of the radioactivity initially present. Thus, all the added precursors, long-chain base, UDP-gal, and ${ }^{14} \mathrm{C}$-acyl-CoA, had been utilized without modification.

Time course and specificity of incorporation of acyl-CoA into ceramide and cerebroside. Incorporation of labeled acyl-CoA into ceramide takes place for only $30-60 \mathrm{~min}$, but incorporation into cerebroside continues for an additional 60-90 min (Fig. 2). Both acyl-CoA's are incorporated into ceramide to a greater extent, and more rapidly, than into cerebroside (note the logarithmic ordinate). Stearate is distinctly better utilized for both syntheses than lignocerate. Al- though not shown, the incorporation of radioactive oleoyl- and palmitoyl-CoA's into ceramide and cerebroside was assayed as above. Incorporation of these acyl-CoA's into ceramide was very low [a result observed previously (5)], and there was almost no incorporation into cerebroside.

Dependence of acyl-CoA incorporation into cerebroside on substrate concentration. Incorporation of radioactive stearoyl- or lignoceroyl-CoA is completely dependent on the presence of UDP-gal (Fig. 3). Cerebroside formation is proportional to long-chain base concentration up to a level of $0.1 \mathrm{mg}$ long-chain base per tube; as the amount of acceptor is increased to $0.6 \mathrm{mg}$ the amount of cerebroside synthesized remains constant. The incorporation of stearoyl-CoA into cerebroside proceeds some $15 \%$ more efficiently if sphingosine, rather than dihydrosphingosine, is the long-chain base acceptor. The results for lignoceroyl-CoA are reversed, dihydrosphingosine being a somewhat more efficient acceptor than sphingosine. This 


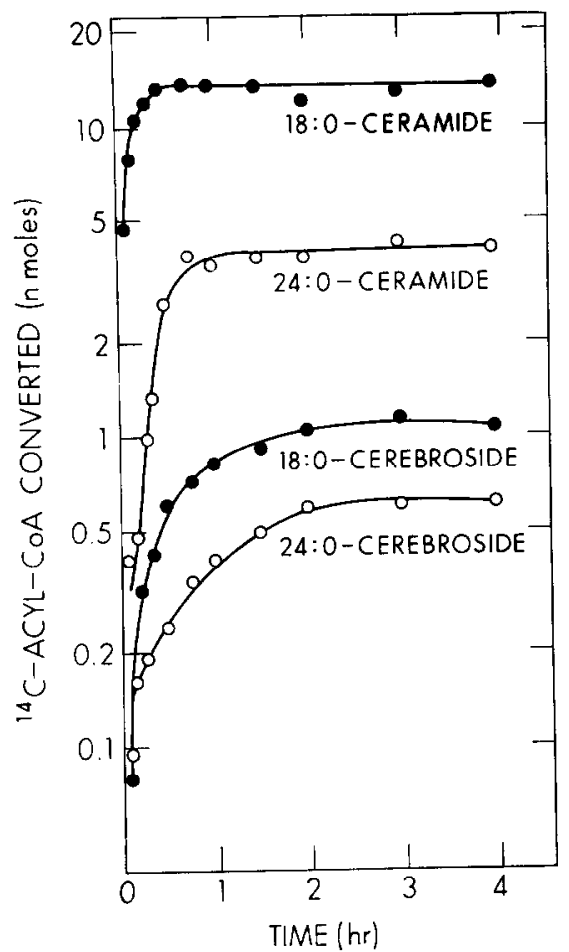

H'IG. 2. Time course of conversion of radioactive acyl-CoA to cerebroside and ceramide. Incubation conditions as described under Methods except that time was varied. The acceptor was dihydrosphingosine. After isolation of total lipids each fraction was divided in two; one aliquot was chromatographed for the identification of cerebroside (as in Fig. 1) and another aliquot was chromatographed in chloroform-acetic acid (9:1) for characterization of ceramide. After radioautography regions corresponding to cerebroside and ceramide were scraped, and radioactivity was determined.

specificity is the same as noted for ceramide formation from radioactive acyl-CoA and long-chain base (5).

Attempts to demonstrate the acylation of psychosine. Incubation mixtures similar to those used in the experiments described above, but substituting psychosine evaporated onto Celite for the long-chain base and omitting UDP-gal, were prepared. Variables studied included (a) addition of varying concentrations of $\mathrm{MgCl}_{2}, \mathrm{MnCl}_{2}$, and/or EDTA; (b) emulsification of psychosine in a variety of detergents, each detergent being tested at several concentra- tions; and (c) use of a variety of buffers in the $\mathrm{pH}$ 6-8 range. No significant incorporation of psychosine into cerebroside was detected. The detergents used were Triton $\mathrm{X}-100$, Tween $20, \mathrm{Na}$ cholate, $\mathrm{Na}$ taurocholate, and MYRJ 59 at concentrations of $0.05 \%$ and $0.2 \%$.

\section{Formation of Cerebroside by Galactosylation of NFA-Ceramide}

Characterization of the product. Utilization of exogenously added ceramide is demonstrated in the radioautogram shown in Fig. 4. The presence of stearoyl sphingosine as an acceptor of labcled galactosc (lane 2) results in formation of 18:0cerebroside while lignoceroyl sphingosine results in formation of 24:0-cerebroside (lane 5). When a mixture of NFA-ceramides prepared from a natural source is used as substrate, synthesis is observed primarily in the long-chain cerebroside regions, although radioactivity is also observed in the medium chain (stearoyl) region (lane 4). This is in accordance with the fatty acid distribution of this sample of ceramide, which is more than $70 \% \mathrm{C}_{22}-\mathrm{C}_{26}$ fatty acids. Quantitative results, obtained by scraping bands (a)

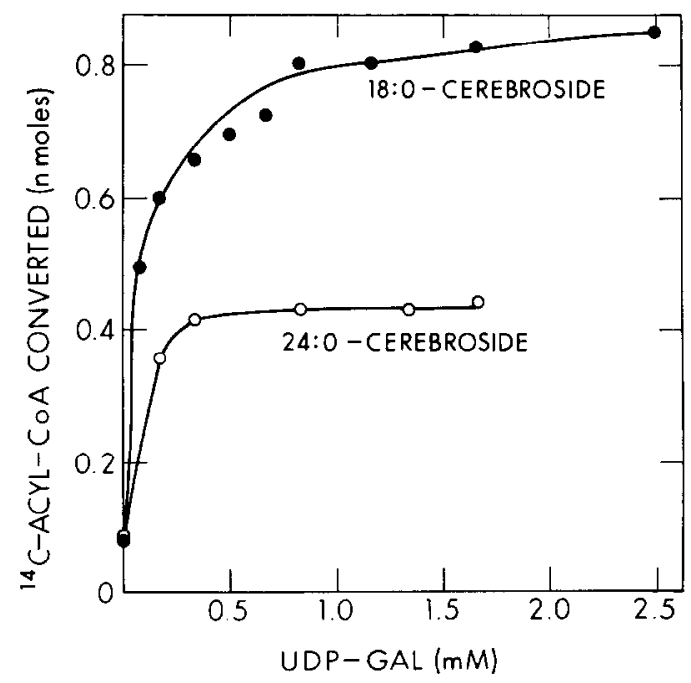

Fig. 3. Conversion of radioactive acyl-CoA to cerebroside as a function of UDP-gal concentration. Incubation conditions as described in Methods except that UDP-gal concentration was varied. 


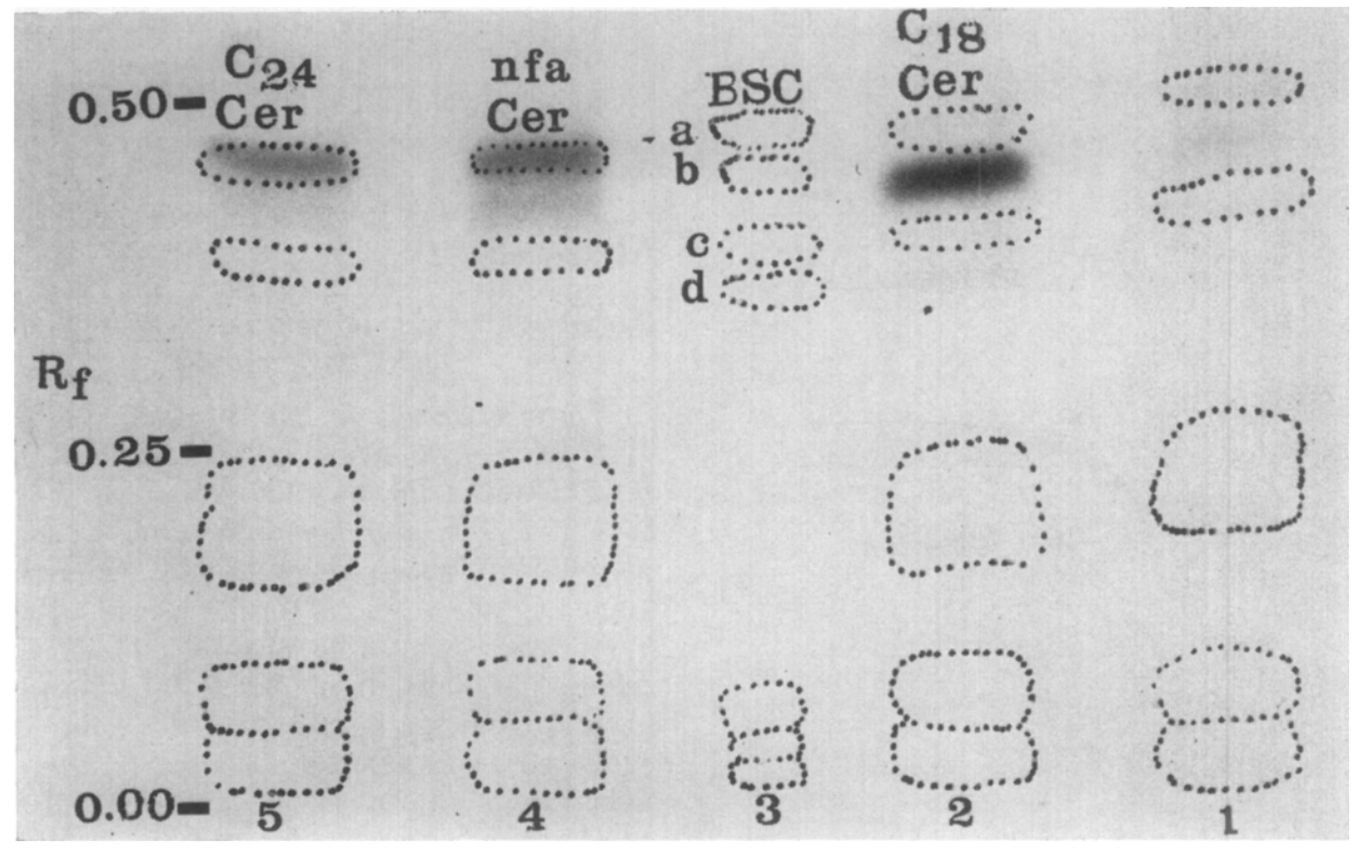

FIG. 4. Formation of radioactive cerebroside from ceramide and radioactive UDP-gal. Incubation conditions as described in Methods. Each tube contained ${ }^{14} \mathrm{C}$-UDP-gal and the following ceramide component (co-evaporated onto Celite with $0.25 \mathrm{mg}$ of lecithin): (1) no acceptor; (2) stearoyl sphingosine; (4) NFA-ceramide prepared from natural sources; (5) lignoceroyl sphingosine. Lipids from each tube were isolated and chromatographed in the corresponding lane as described in Methods. Lane 3 contained standard sphingolipids. For meaning of stippled lines refer to Fig. 1 .

and (b) (Fig. 4) and carrying out radioactivity determination with several such TLC plates, are shown in Table I. It can be seen that in control incubations (ceramide omitted), incorporation into the stearoyl cerebroside region is greater than incorporation into the long-chain cerebroside region. In further expcriments, only incorporation into the long-chain cerebroside region was assayed in order to increase the reliability of the assay with respect to stimulation above background.

In another experiment, not shown, synthetic stearoyl sphingosine and stearoyl dihydrosphingosine were used as substrates. The labcled galactosyl stearoyl dihydrosphingosine formed moved distinctly ahead of the galactosyl stearoyl sphingosine. In each of the above experiments samples were eluted, stability to alkaline methanolysis was demonstrated, and the cerebrosides were run on borate TLC plates to rule out conversion to glucocerebroside.

As a final check to demonstrate that the experimental conditions result in incorporation of exogenously added ceramide, an incubation was conducted with radioactive lignoceric ceramide as substrate. Conditions were the same as in Fig. 4, except that $0.3 \mu$ mole of unlabeled UDP-gal was substituted for the ${ }^{14} \mathrm{C}-\mathrm{UDP}$-gal, and ${ }^{14} \mathrm{C}$ lignoceroyl $\mathrm{D}$-sphingosine $(50$ nmoles at $7500 \mathrm{dpm} /$ nmole) was substituted for the unlabeled ceramide. A control incubation was carried out with the omission of UDPgal. Radioactivity incorporated into the long-chain cercbroside region in the cxperimental incubation corresponded to 0.12 nmoles; radioactivity in the cerebroside region from the control incubation was 0.035 nmoles.

Phospholipid requirement for cerebroside 
TABLE I

Specificity of the Ceramide Substrate for Formation of Cerebroside or GLUCOCEREBROSIDE ${ }^{a}$

\begin{tabular}{l|c|c|c}
\hline \multicolumn{1}{c|}{ Acceptor } & \multicolumn{2}{|c}{$\begin{array}{c}\text { Cerebroside } \\
\text { formed } \\
\text { (pmoles) }\end{array}$} \\
\cline { 2 - 4 } & $\begin{array}{c}\text { 14C } \\
\text { nucleotide } \\
\text { sugar }\end{array}$ & $\begin{array}{c}\text { Me- } \\
\text { dium } \\
\text { chain }\end{array}$ & $\begin{array}{c}\text { Long } \\
\text { chain }\end{array}$ \\
\hline No acceptor & UDP-gal & 37 & 20 \\
Stearoyl sphingosine & UDP-gal & 96 & 27 \\
Lignoceroyl sphingosine & UDP-gal & 44 & 68 \\
NFA-ceramides & UDP-gal & 62 & 94 \\
No acceptor & UDP-gle & 140 & 90 \\
Stearoyl sphingosine & UDP-glc & 720 & 100 \\
Lignoceroyl sphingosine & UDP-glc & 190 & 560 \\
NFA-ceramides & UDP-glc & 740 & 820 \\
\hline
\end{tabular}

a The ceramide indicated $(0.25 \mathrm{mg})$ was coevaporated with crude lecithin, the specified radioactive nucleotide sugar and other components added, and incubation carried out as described in Methods. The appropriate carrier was added, the lipids were separated by TLC, and radioactive bands corresponding to mediumand long-chain NFA-galactocerebrosides or glucocerebrosides collected separately and counted. Results shown are the average of three experiments.

synthesis. For ceramide to be efficiently incorporated into cerebroside under our experimental conditions, it was necessary to coevaporate the NFA-ceramide and lecithin onto Celite (Fig. 5). It can be seen that the crude lecithin also stimulated the blank reaction, the utilization of endogenous NFA-ceramide.

Time course of cerebroside formation from ceramide. Incorporation of ${ }^{14} \mathrm{C}-\mathrm{UDP}$-gal is linear with time for at least three hours (Fig. 6). When data for formation of the long-chain NHA-cerebroside are examined (Fig. 6a) it can be seen that endogenous precursor is present at low levels and is used up within the first hour. At $4 \mathrm{hr}$, incubation tubes with exogenous ceramide have formed five times as much ccrcbroside as control tubes without ceramide. When incorporation into the medium chain cerebroside fraction is studied (Fig. 6b) it can be seen that incorporation due to endogenous precursor is considerable. Incorporation of endogenous

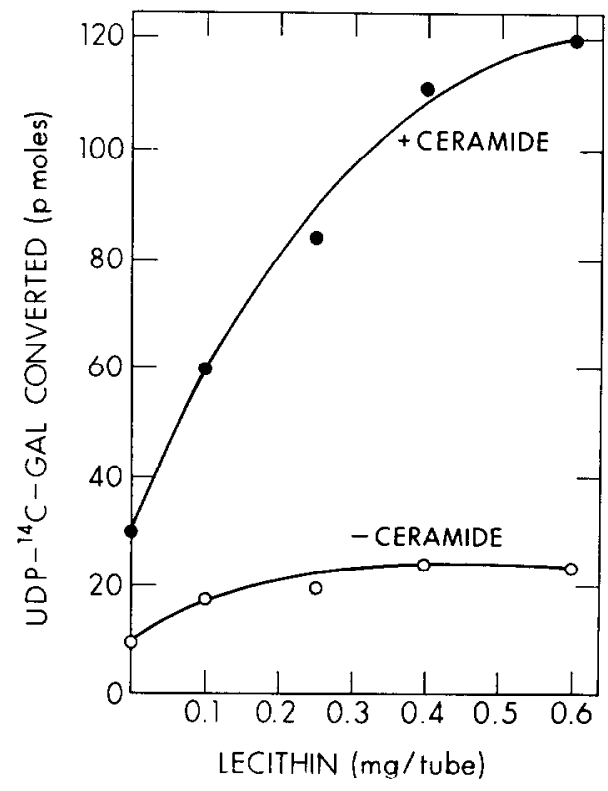

Frg. 5. Conversion of radioactive UDP-gal to cerebroside as a function of the amount of phospholipid present. Conditions as described in Methods except that the amount of lecithin coevaporated with $0.25 \mathrm{mg}$ of mixed NFA-ceramides on the Celite was varied as indicated. In control tubes the NFA-ceramide was omitted. After isolation of lipids, TLC, and radioautography, the radioactive band corresponding to long-chain cerebroside was scraped, and the radioactivity was determined.

precursor does not level off until $2 \mathrm{hr}$. These data, as well as the observation that the ceramides of whole brain contain primarily medium chain $\mathrm{NFA}^{3}(25)$, indicate that our microsomal fractions contained some endogenous NFA-ceramide. This hypothesis was confimed in a qualitative manner by extracting total lipids from a quantity of microsomes and assaying for trace amounts of ceramide by heavily overloading a TLC plate. A small amount of ceramide, primarily stearoyl ceramide, was observed.

Dependence of cerebroside formation on substrate and enzyme concentration. The synthesis of cerebroside is dependent on the amount of NFA-ceramide available as substrate (Fig. 7) and the concentration of microsomal protein (Fig. 8).

${ }^{3}$ Unpublished work with whole mouse brain, Antoinette Brenkert and N. S. Radin. 


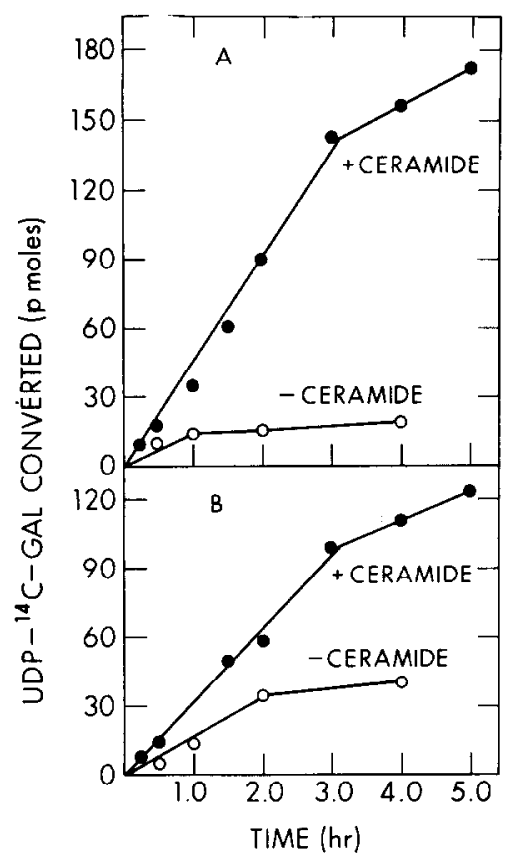

Fig. 6. Time course of conversion of UDP-gal to cerebroside. Conditions as in Methods except that time was varied. In control tubes only the mixture of NFA-ceramides was omitted. After isolation of lipids, TLC and radioautography the radioactive bands corresponding to medium- and long-chain NFA-cerebrosides were collceted separately and radioactivity determined. (a) Results for medium chain cerebrosides. (b) Results for long chain cerebrosides.

Formation of glucocerebroside by glucosylation of NFA-ceramide. Table I also indicates the results obtained when ${ }^{14} \mathrm{C}$-UDP-glc is substituted for radioactive UDP-gal. Stearoyl and lignoceroyl sphingosine produce 18:0 and 24:0-glucocerebroside, respectively. The results for formation of glucosyl ceramide parallel those for galactosyl ceramide formation, except that incorporation of glucose into glucocerebroside is considerably more efficient than the corresponding reaction involving galactose. TLC on borate plates confirmed the identification of the glucocerebroside.

It is of interest to note that lignoceroyl sphingosine is a good substrate for glucosylation. This ceramide is probably not a physiological substrate since brain forms glucocerebroside primarily as a precursor to gangliosides, which do not contain any $\mathrm{C}_{24}$
$N$-acyl fatty acids. These data support the original observation of Basu et al. (6) that exogenous ceramide stimulates synthesis of glucocerebroside by chick brain microsomes

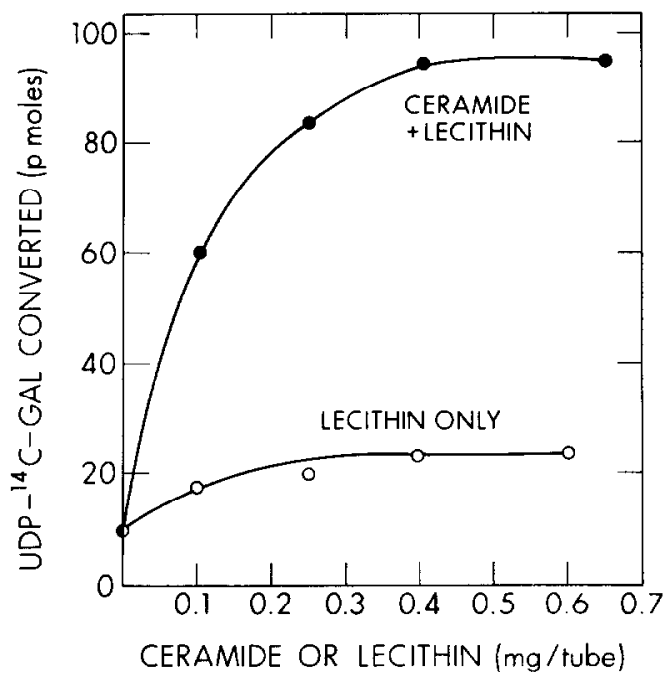

Fig. 7. Conversion of radioactive UDP-gal to cerebroside as a function of the amount of mixed NFA-ceramides present. Incubation conditions were as described in Methods except that the amount of ceramide was varied as indicated. With increasing ceramide concentration the amount of crude lecithin present was also increased, the lecithin/ceramide ratio being kept at 1 on a wt/wt basis. Only the radioactivity incorporated into long chuin NFA-cerebroside has been plotted.

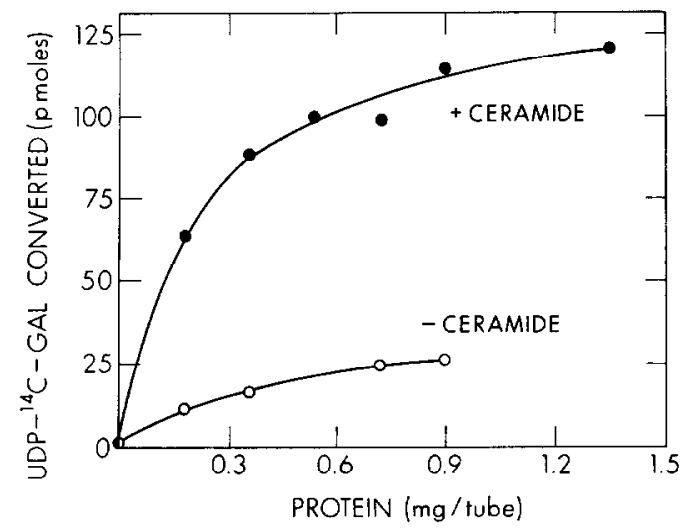

FIg. 8. Conversion of radioactive UDP-gal to cerebroside as a function of microsomal protein concentration. Incubation conditions as described in Methods (with mixed NFA-ceramide as acceptor) except that the amount of microsomal protein was varied as indicated. Long-chain cerebrosides only are plotted. 
Our radioautographic data furnish additional evidence that the exogenous ceramide is actually utilized as a substrate and does not simply stimulate the utilization of endogenous ceramide.

\section{DISCUSSION}

In a previous publication detailing the evidence that HFA-ceramide is a precursor to HFA-cerebroside ( 7 ) we were unable to show stimulation of NFA-cerebroside formation by addition of NFA-ceramide to a cellfree assay system. Now, by modification of the incubation conditions and use of an improved assay we are able to demonstrate this reaction. The increased sensitivity of the assay is obtained by use of a TLC system which separates the desired product from the nonspecific background caused by incorporation of radioactive galactose into other lipids.

It is not yet evident whether two different enzymes are involved in the synthesis of NFA- and HFA-cerebrosides or whether HFA-ceramide is simply a much better galactose acceptor. Mammalian brain contains more HFA-cerebroside than NFAcerebroside (26) but the difference is small. Perhaps the factor controlling the rate of deposition of each group of cerebrosides is the rate of synthesis of each type of ceramide. We have been unable to detect HFA-ceramide in brain ${ }^{3}$ although NFAceramide is readily discerned. Thus, it secms possible that the rate-limiting step in HFAcerebroside deposition is formation of HFAceramide, while the rate-limiting step in NFA-cerebroside deposition is the galactosylation reaction.

Fujino and Nakano (27) have recently published a note on the biosynthesis of cerebroside from ceramide by rat brain microsomes, but it is not clear whether the biosynthetic cerebroside was of the hydroxy or nonhydroxy type. Moreover, it was not determined whether the biosynthetic cerebroside was actually derived from the exogenous ceramide mixture or whether the exogenous ceramide-detergent mixture simply stimulated the galactosylation of endogenous material. We made several attempts at reproducing these results with synthetic stearoyl or lignoceroyl sphingosine as substrate, and using incubation conditions identical to those reported (27). We confirmed the increased cerebroside formation on addition of emulsified NFA-ceramide, and showed that the product was indeed NFA-cerebroside, but a control with Tween 20 (one of the detergents in the emulsion) yielded a similar effect. Thus, it is likely that the increase in cerebroside formation observed by Fujino and Nakano was due to the detergent component of the mixture. It is not clear from their paper whether they ran a control incubation with detergent alone. Some other preliminary reports suggesting the possibility that ceramide is precursor to cerebroside have appeared (1, $28,29)$.

Three types of evidence point strongly to the involvement of exogenous NFA-ceramide as the actual galactose acceptor: (a) We have demonstrated, by TLC and radioautography, that a medium-chain ceramide (stearoyl sphingosine) gives rise to a mediumchain cerebroside, and a long-chain ceramide (lignoceroyl sphingosine) gives rise to a long-chain cerebroside. Similar discrimination between precursor and product was made on the basis of the long-chain base component of the ceramide acceptor (stearoyl sphingosine and stearoyl dihydrosphingosine). (b) We have demonstrated direct conversion of radioactive lignoceroyl sphingosine to long-chain NFA-cerebroside. (c) We have evidence for involvement of ceramide from examination of the system which utilizes all three precursors, acylCoA, UDP-gal, and long-chain base. There is a parallelism between the rates of ceramide formation and the rates of cerebroside formation, as a function of the fatty acid and long-chain base used. Moreover, our observation (Fig. 2) that acyl incorporation continues for a longer period of time into cerebroside than into ceramide is consistent with the role of the latter as precursor of the former.

It is interesting to note that our data do not supply any evidence that the glycosylation of ceramides has specificity based on the chain length of the $N$-acyl fatty acid moiety. This is in contrast to the formation of NFA-ceramide, via acylation of longchain base, which exhibits high specificity 
for the chain length of the acyl-CoA substrate. This suggests that the factor determining the fatty acid distribution within the naturally occurring NFA-cerebrosides and gangliosides is the rate of synthesis of each NFA-ceramide, and its availability to the glycosylating enzyme.

We directed considerable effort towards demonstration of an enzymatic activity for the acylation of psychosine, a possible alternative route for formation of cerebroside. We feel that our negative results are significant, since our incubation conditions contained all the enzymatic activities required for biosynthesis of NFA-cerebroside from its primary components, long-chain base, galactose, and fatty acid. It is likely that a previous report (3) indicating stimulation of radioactive acyl-CoA incorporation into cerebroside by psychosine reflects a nonspecitic increase in lipid synthesis due to addition of psychosine. This hypothesis is supported by the observation that, in a rat brain homogenate, addition of psychosine causes a 7 -fold increase in radioactive acetate incorporation into the NFA of various lipids. ${ }^{4}$ Kanfer also has observed the nonacylation of psychosine (30), but his experiments were carried out with palmitoyl-CoA, which we have found to be a poor donor in NFA-ceramide and cerebroside synthesis.

Other alternative routes for the biosynthesis of NFA-cerebroside, such as acylation of ketodihydrosphingosine (5) followed by galactosylation and reduction of the keto group, or formation of HFAcerebroside followed by reduction of the HFA moiety, are possible. However, at the present time the available evidence indicates that galactosylation of NFA-ceramide is the major route for biosynthesis of NFAcerebroside.

\section{ACKNOWLEDGMENT}

We thank Mrs. Carolyn Seidl and Mrs. Inez Mason for technical assistance.

\section{REFERENCES}

1. Burton, R. M., Sodd, M. A., and Brady, R. O., J. Biol. Chem. 233, 1053 (1958).

${ }^{4}$ Unpublished work, A. K. Hajra, K. C. Kopaczyk, and N. S. Radin.
2. Clemand, W. W., and Kennedy, E. P., $J$. Biol. Chem. 235, 45 (1960).

3. Brady, R. O., J. Biol. Chem. 237, 2416 (1962).

4. Sribnex, M., Biochim. Biophys. Acta 125, $542(1966)$.

5. Morell, P., and Radin, N. S., J. Biol. Chem. 245, 342 (1970).

6. Basu, S., Kaufman, B., and Roseman, S., J. Biol. Chem. 243, 5802 (1968).

7. Moneld, P., and RadiN, N. S., Biochemistry 8, 506 (1969).

8. Basu, S., Schultz, A., and Basu, M., Fed. Proc. 28, 540 (1969).

9. Coles, L., and Gray, G. M., Biochem. Biophys. Res. Commun. 38, 520 (1970).

10. Braun, P. E., Morell, P., and Radin, N. S., J. Biol. Chem. 245, 335 (1970).

11. Young, D. L., And Lynen, F., J. Biol. Chem. 244, 377 (1969).

12. Kadin, N. S., ANd Brown, J. R., Biochem. Prep. 7, 31 (1960).

13. Carter, H. E., Rothfus, J. A., and Gigg, R., J. Lipid Res. 2, 228 (1961).

14. KopaczYK, K. C., and RadtN, N. S., J. Lipid Res. 6, 140 (1965).

15. HaJra, A. K., ANd Radin, N. S., J. Lipid Res. 1. 4, 448 (1963).

16. GATT, S., Biochim. Biophys. Acta 70, 370 (1963).

17. Bergström, S., PaAbo, K., and Rottenbera, M., Acta Chem. Scand. 6, 1127 (1952).

18. Barenholz, Y., and GatT, S., Biochim. Biophys. Acta 152, 790 (1968).

19. RAdin, N. S., Chemist-Analyst 55, 117 (1966).

20. Suomi, W. D., and Agranoff, B. W., J.Lipid Res. 6, 211 (1965).

21. KEAN, F. I., I. Iipid Res. 7, 449 (1966).

22. Young, O. M., and Kanfer, J. N., J. Chromatogr. 19, 611 (1965).

23. Kishinoto, Y., Davies, W. E., and Radin, N. S., J. Lipid Res. 6, 525 (1965).

24. Kishimoto, Y., and Radin, N. S., J. Lipid Res. 6, 435 (1965).

25. O'Brien, J. S., And Sampson, E. L., J. Lipid Res. 6, 545 (1965).

26. Kishimoto, Y., and Radin, N. S., J. lipid Res. 1, 79 (1959).

27. Fujino, Y., and Nakano, Mo., Biochem. $J$. 113, 573 (1969).

28. Radin, N. S., in "The Biology of Myelin" (S. R. Korey, ed.), p. 271. Harper, New York (1959).

29. Burton, R. M., in "Lipids and Lipidoses" (G. Schettler, ed.) p. 122. Springer, Berlin (1967).

30. KANFER, J. N., Lipids 4, 163 (1969). 